\title{
Affection of Cranial Nerves in COVID-19 Patients Should Prompt Suspicion of Guillain-Barre Syndrome
}

\author{
Josef Finsterer $^{a}$ Fulvio A. Scorza ${ }^{b}$

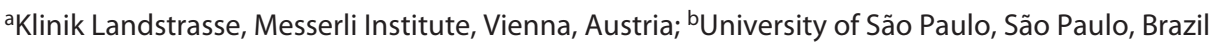

\section{Dear Editor,}

We read with interest the article by Decavel et al. [1] about a retrospective study of 10 patients admitted to the post-ICU unit after having been treated between 8 to 40 days on the ICU who presented on admission with affection of a single or multiple lower cranial nerves. The cause of lower cranial nerves palsies was attributed to pressure palsies due to the bedding of these patients on the ICU during artificial ventilation [1]. It was concluded that bedding on the ICU should be improved by application of adapted cushions to control the position of the head during prone position ventilation [1]. The study is appealing but raises several comments and concerns.

We do not agree with the notion that cranial nerve lesions in the 10 included patients were due to pressure palsies. Various differentials were not appropriately excluded.

The most important differential diagnosis not excluded is Guillain-Barre syndrome (GBS) with or without involvement of the cranial nerves or the GBS subtype monoor polyneuritis cranialis. SARS-CoV-2-associated GBS with or without involvement of the cranial nerves is increasingly recognized as a complication of COVID-19. As per the end of December 2020, at least 220 patients with SARS-CoV-2-associated GBS have been reported [2] and as per the end of July 2021, at least 300 patients with SARS-CoV-2-associated GBS were collected [Finsterer, submitted].

To exclude or confirm GBS, application of the Brighton criteria is crucial. GBS with or without involvement of cranial nerves has been repeatedly reported in SARSCoV-2-infected patients [3]. Thus, a limitation of the study is that none of the 10 included patients underwent a spinal tap and investigations of the cerebrospinal fluid or nerve conduction studies (NCSs) [1]. Suspecting GBS is justified as at least seven of the 10 included patients had weakness of limb muscles according to Table 1 [1].

Another limitation of the study is that none of the included patients underwent NCSs. Particularly, if cranial nerves VII, VIII, or XII are affected, NCSs are feasible and could provide information if these nerves are affected not only clinically but also electrophysiologically. Concerning cranial nerve VIII, it could be helpful to apply acoustically evoked potentials to assess if conduction of cochlear impulses was disturbed or not. Information of NCSs is crucial for application of the Brighton criteria.
Correspondence to:

Josef Finsterer, fifigs1@yahoo.de 
Since one of the subtypes of GBS is Bickerstaff encephalitis, which goes along with involvement of the lower cranial nerves, it is crucial that all 10 patients had undergone imaging of the brain by MRI with contrast medium. Cerebral MRI is also crucial for excluding immune encephalitis of the brainstem. Furthermore, cerebral MRI with contrast medium is helpful for documenting the affection of cranial nerves as they can show T2 hyperintensities or gadolinium enhancement along the course of the nerve [3]. A second differential not appropriately excluded was critical ill neuropathy.

Since all cranial nerves can be affected in COVID-19 [3], we should know why affection of cranial nerves I to VI was not included in the evaluation. Hyperintensities of the olfactory bulb have been reported [4], optic neuritis has been reported [5], and affection of cranial nerves III-VI was encountered [3].

We should be informed about the treatment the $10 \mathrm{pa}-$ tients received for their cranial nerve palsies. How many of the patients received steroids, immunoglobulins, or even plasmapheresis?

According to Table 1, 4 patients did not significantly improve with regard to the MRC-SS score [1]. We should be told which treatment these 4 patients received and if there is a plausible explanation why they did not recover.
Overall, the study has several limitations which challenge the results and their interpretation. These issues should be addressed not to draw unsupported conclusions.

\section{Statement of Ethics}

Ethical approval and consent to participate: not applicable. Consent for publication: not applicable. Availability of data and material: all data reported are available from the corresponding author.

\section{Conflict of Interest Statement}

None.

\section{Funding Sources}

None received.

\section{Author Contribution}

J.F.: design, literature search, discussion, first draft, critical comments. F.A.S.: literature search, discussion, critical comments, and final approval.

\section{References}

1 Decavel P, Nahmias O, Petit C, Tatu L. Lower cranial nerve palsies in the COVID-19 pandemic: a 10-case series of intensive care unit patients. Eur Neurol. 2021 Sep 23:1-4.

2 Finsterer J, Scorza FA. Guillain-Barre syndrome in 220 patients with COVID-19. Egypt J Neurol Psychiatr Neurosurg. 2021;57(1):55.
3 Finsterer J, Scorza FA, Scorza C, Fiorini A. COVID-19 associated cranial nerve neuropathy: a systematic review. Bosn J Basic Med Sci. 2021 Aug 11.

4 Kim PH, Kim M, Suh CH, Chung SR, Park JE, Kim SC, et al. Neuroimaging findings in patients with COVID-19: a systematic review and meta-analysis. Korean J Radiol. 2021 Nov;22(11):1875-85.
5 Corrêa DG, Hygino da Cruz LC Jr, Lopes FCR, Rangel CC, de Araújo Henriques Tessarollo AL, Coelho KCG, et al. Magnetic resonance imaging features of COVID-19-related cranial nerve lesions. J Neurovirol. $2021 \mathrm{Feb}$; $27(1): 171-7$. 